\title{
On the effects of MPC on a domestic energy efficiency optimization methodology
}

\author{
Albert Molderink, Vincent Bakker, , Maurice G.C. Bosman Johann L. Hurink, Gerard J.M. Smit \\ University of Twente, Department of Computer Science, Mathematics and Electrical Engineering \\ P.O. Box 217, 7500 AE, Enschede, The Netherlands \\ a.molderink@utwente.nl
}

\begin{abstract}
Increasing energy prices and the greenhouse effect lead to more awareness of energy efficiency of electricity supply. During the last years, a lot of technologies and optimization methodologies were developed to increase the efficiency, maintain the grid stability and support large scale introduction of renewable sources. In previous work, we showed the effectiveness of our three-step methodology to reach these objective, consisting of 1) offline prediction, 2) offline planning and 3) online scheduling. Although initial results are promising, one of the problems of the current implementation is the inability to work around prediction errors in the last step. Therefore, we added Model Predictive Control to step three to incorporate future states in the control to work around prediction errors. Adding MPC improves the ability to work around prediction errors and especially improves the irregular behavior of devices, resulting in a more stable situation.
\end{abstract}

Keywords: Micro-generation, Energy efficiency, Microgrid, Virtual Power Plant, Smart grid, Model Predictive Control

\section{INTRODUCTION}

In last couple of decades ever more attention has been directed towards electricity supply and infrastructure. On the one hand, electricity consumption increased significantly and became very fluctuating. Since the maximum peak consumption defines the generation and grid capacity, the required capacity has increased. Furthermore, due to the fluctuations in consumption (and therefore in required generation) the generation efficiency decreased [1].

On the other hand, reduction in the $\mathrm{CO}_{2}$ emissions and introduction of generation based on renewable sources are important topics today. However, these renewable resources are mainly given by very fluctuating and uncontrollable sun, water- and wind-power. The generation patterns resulting from these renewable sources may have some similarities with the electricity demand patterns, but they are not equal. For this reason, supplemental production is required to keep the demand and supply in balance, resulting in an even more fluctuating generation pattern for the conventional power plants. Finally, the introduction of new, energy efficient technologies such as electrical cars can result in an even further fluctuating electricity demand. If electrical cars are charged in an uncontrolled way, this may result in high peak demands of electricity since these vehicles often will be charged in the evening and need to be charged fast to ensure enough capacity for the upcoming trip. Lowering the peaks in demand is desirable to improve the utilization of the available grid capacity.

A solution for these problems may be to transform domestic customers from static consumers into active players in the production process. More and more new technologies with controllable load and generation are developed, such as controllable white goods and micro-generation. Furthermore, domestic energy storage of both heat and electricity is becoming quite common. The goal of our research is to determine a methodology to use this optimization potential to 1) optimize efficiency of current power plants, 2) support the introduction of a large penetration level of renewable sources (and thereby facilitate the means that are needed for $\mathrm{CO}_{2}$ reduction) and 3 ) optimize utilization of the current grid capacity.

In [2] a control strategy is presented to exploit this optimization potential in a generic way. The methodology is flexible in both the optimization objective and the technologies available within houses. After all, objectives may differ over time and different houses may have different technologies installed. This control strategy consists of three steps.

In the first step, a system located at the consumers predicts the production and consumption pattern for all appliances for the upcoming day. For example, in a normal household multiple appliances like a tv, washing machine, central heating are present. For each appliance, based on the historical consumption pattern of the residents and external factors like the weather, a predicted energy profile is generated. Based on the expected energy profile and the characteristics of the devices the scheduling freedom and optimization potentials are determined. These potentials are aggregated by the local controller and sent to the global controller. The global controller is structured as a hierarchical tree for scalability and to reduce communication. In each node of the tree the received profiles are aggregated and sent upwards in the tree until the root node. In the second step, these optimization potentials can be used by a central planner to exploit the potential to reach a global objective. The root node determines steering signals based on the received information and the objective. These steering signals are distributed via the tree structure, whereby each node may adjust the steering signals. Adjusted profiles are determined in the houses, based on the (new) steering signals and the predictions. These new profiles are again 
send upwards. In this iterative way a near-optimal solution can be found with a reasonable computational time. Example objectives are peak shaving or compensating the fluctuation of the production of renewable sources like wind-parks. The result of the second step is a planning for each household for the upcoming day and a overall production/consumption profile. In the final step, which is the focus of this paper, a realtime control algorithm decides at which times appliances are switched on/off, when and how much energy flows from or to the buffers and when and which generators are switched on. This realtime control algorithm uses the steering signals from the global planning as input, but preserves the comfort of the residents in conflict situations. The local controller can also run independently, for example when the connection with the global controller is lost.

One of the drawbacks of the current implementation of this approach is that the planning is based on predictions and therefore the planning often cannot be reached. A small prediction error can result in large deviations from the planning since the realtime controller does not have a look ahead feature, but locally tries to follow the planning [2]. More general, since the realtime controller only takes the current status of the system into account it may take decisions that are disadvantuous for later time periods. Therefore, in this paper a method is studied to improve the realtime controller such that it not only takes the current status into account, but also a number of future states, based on improved shortterm predictions. In this way, it might be possible to prevent disadvantuous decisions and to work around prediction errors. Furthermore, since a larger horizon is observed, it can be determined earlier when the prediction errors are too large and a new planning need to be determined (step 1 and step 2).

The rest of this paper is structured as follows. The next section describes the general approach and the used models. Section III describes the local control methodology and next in Section IV the principle of Model Predictive Control (MPC) is described together with a way to incorporate it in the algorithm. In Section V an implementation of smart appliances with MPC is described and Section VI discusses simulations and results. Section VII ends up with conclusions.

\section{APPROACH}

To analyze the energy-streams and optimization potential, a general modelling of the energy situation in a domestic environment has been set up. The basis of this modelling is the model of a house. Since the behavior of individual devices is optimized, the detail level of the model is on the device level. Houses contain multiple devices and exchange energy with the environment (e.g. gas import, electricity import/export) and multiple houses can be combined in a grid to analyze their overall behavior. Based on this model, a simulator is built to be able to quickly simulate different scenarios, house configurations and device parameters [3]. An example of a model of a house is shown in Figure 1.

Multiple types of energy can flow through the house (e.g. gas, electricity, heat). These types of energy are modelled

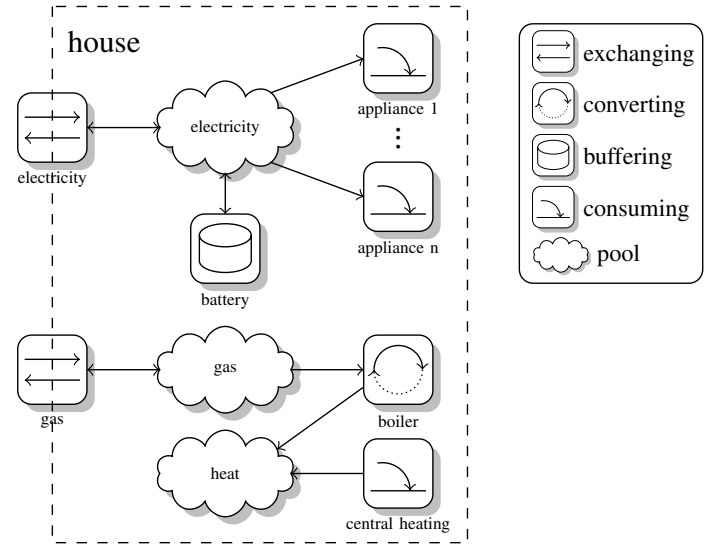

Fig. 1. Model of the house

as streams transporting one type of energy. These energy-types are converted, buffered and consumed by devices. Furthermore, energy-types can be exchanged with the environment, which is modelled by exchanging devices. Every device can have certain energy-streams flowing in and certain energystreams flowing out, e.g. a microCHP has a gas stream in and an electricity and a heat stream out.

Energy flows between devices, i.e. the energy-streams of the devices are connected with each other. Sometimes the energy flows directly from one device to one other device (e.g. heat from the boiler to the central heating) while in other cases energy can flow from and to multiple devices (e.g. electricity). Therefore, pools are introduced. Each energy-stream from the devices is connected to a pool. One or more energy-streams can flow into the same pool and one ore more energy-streams can flow out of the same pool. Since a discrete simulation is used, the simulation horizon is discretisized resulting in a set of consecutive time intervals. Every time interval the pools in the house need to be in balance, i.e. as much energy must flow into the pool as flows out. A detailed description of the model and the simulator can be found in [3].

The balance in the pools can be reached, both in the simulation as in real-world scenarios, by using the flexibility of devices: some devices can vary the amount of energy flowing in and/or out. For example, a boiler can be switched on or off, the amount of electricity imported from the grid can vary, a certain amount of energy can be stored or supplied by a buffering device and some consuming devices can be shifted in time. The decisions influence the energy efficiency, electricity import profile, etc. and therefore some decisions are more desirable than others. The goal of the local controller is to make good decisions given a certain objective (e.g. peak shaving or following a global objective). The local controller can work independently or cooperating in the global three step methodology. The steering signals from the global controller are incorporated as energy import/export prices. When a local optimization is used, the objective is also incorporated using the energy import/export prices. The control algorithm used for 
this model is based on the control algorithm described in [4], a detailed description of the algorithm is given in Section III.

The local control methodology can be extended by Model Predictive Control (MPC) [5](in the Operation Research literature this is called Rolling Horizon (RH) [6]). The idea of MPC is to take a number of future time intervals into account while making a decision, using predictions of the future states. A detailed description of this extension is given in Section IV. In this paper the influence of incorporating future time intervals on the control methodology is analyzed, both with and without a global planning.

To analyze the effect of adding this extension, two case studies are simulated. In the first use case the runtime of a freezer in a single house is optimized, with and without MPC. The objective is to minimize the electricity costs with as challenge to work around prediction errors. With this case study the effect on binary devices (on/off) with an internal state can be studied. In [7] MPC is used for short-term scheduling of thermal household devices to decrease balancing costs of wind turbines, i.e. reacting on fluctuations in generation. No offline planning is used and the MPC algorithm is implemented on a global level. The described methodology decreased the imbalance with $33 \%$.

The second use case is based on the use case described in [8]. In that paper the runtime of a microCHP device in 39 houses is optimized and concluded that the threestep methodology is not capable of dealing with prediction errors. In this paper the last step is extended with MPC to analyze whether MPC improves the capabilities to work around prediction errors.

\section{LOCAL CONTROL METHODOLOGY}

Based on the model described above, the goal of the control methodology is to use the flexibility of the devices in such a way that the energy-streams within the house are in balance while working towards an objective. In this section the control methodology during one time interval is described, so this algorithm is executed every time interval.

Every house has a set of devices $D$ and a set of pools $P$. Depending on how a device $d \in D$ is used during the considered time interval, it will lead to a certain internal energy flow $x_{d}$. All streams in and out of device $d$ are connected to a pool $p \in P$ and the amount of energy flowing through a stream is a factor of $x_{d}\left(M_{d p} \times x_{d}\right)$ (5). The multiplication factors $M_{d p}$ must be defined correctly, meaning that all energy is preserved [3]. The pools are in balance when the amount of energy flowing in and out is equal (5). By definition, the energy-streams towards pools are negative and streams from the pools are positive.

The flexibility of a device is expressed in the allowed values for $x_{d}$. For example, a consuming device can be switched on $\left(x_{d}=\right.$ demand $)$ or off $\left(x_{d}=0\right)$. The grid (exchanger) can import/export a certain amount of electricity (e.g. $-2000 \mathrm{~kW} \leq x_{d} \leq 5000 \mathrm{~kW}$ ). The possible values for $x_{d}$ are expressed using a set of intervals $I_{d}$, where every interval $i \in I_{d}$ is specified by its lower and upper bound

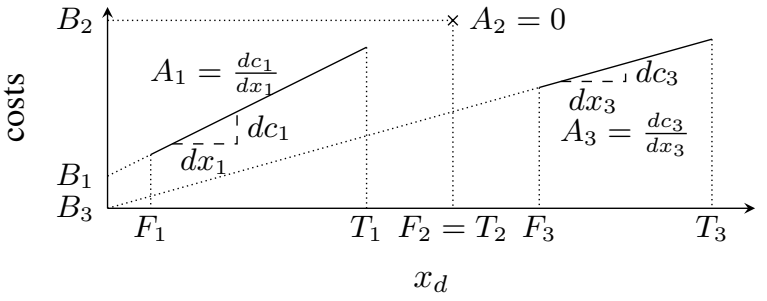

Fig. 2. Example valid range for $x_{d}$ and corresponding costs

$\left(F_{d, i}\right.$ and $\left.T_{d, i}\right)$. An example of such a set of intervals is shown on the horizontal axis in Figure 2. The value of $x_{d}$ must be chosen on one of the intervals. Therefore, for every interval a binary variable $c_{d, i}$ is introduced. Only one of these variables can be nonzero (8) and $x_{d}$ is chosen from the corresponding interval (3),(4). The multiplication factors $M_{d p}$ can also depend on the chosen interval, e.g. due to differences in efficiency. Therefore, for every interval $i \in I_{d}$ multiplication factors $M_{d p, i}$ are defined, $M_{d p}$ depends on the chosen interval (6). All devices are independent and therefore the valid range of every $x_{d}$ is also independent.

Some decisions are more preferable than others for the residents, e.g. temporarily switching off a television is less desirable than temporarily switching off the freezer. Furthermore, switching on and off a device often can lead to wearing. Finally, the amount of electricity imported or exported is topic of desirability, depending on the objective. These preferences can be expressed using cost functions. The cost functions have the same structure for every device $(A \times x+B)$ and the cost functions can differ per interval $i \in I_{d}$ leading to a total cost $t c_{d}$ for each device (2). An example of the combination of valid intervals for $x_{d}$ and corresponding costs is shown in Figure 2.

Given the balancing constraints, the possible consumption/production values for every device $\left(x_{d}\right)$ and the cost function, the optimization algorithm searches the solution with the lowest costs:

$$
\begin{gathered}
\sum_{d \in D} t c_{d} \\
\text { s.t. } \quad t c_{d}=\sum_{i \in I_{d}} A_{d, i} \times x_{d, i}+B_{d, i} \times c_{d, i}, \forall d \in D \\
c_{d, i} \times F_{d, i} \leq x_{d, i} \leq c_{d, i} \times T_{d, i} \forall i \in I_{d}, \forall d \in D \\
x_{d}=\sum_{i \in I_{d}} c_{d, i} \times x_{d, i}, \forall d \in D \\
\sum_{d \in D} M_{d p} \times x_{d}=0 \forall p \in P \\
M_{d p}=\sum_{i \in I_{d}} M_{d p, i} \times c_{d, i} \forall d \in D, \forall p \in P \\
c_{d, i} \in\{0,1\} \forall i \in I, \forall d \in D \\
\sum_{i \in I_{d}} c_{d, i}=1 \forall d \in D
\end{gathered}
$$

With this control methodology all domestic appliances can be modelled, smart controllable devices but also conventional 
appliances. A conventional television only has one valid value for $x_{d}$ while a smart freezer and a microCHP/heat buffer combination can have several options for $x_{d}$ from which a choice can be made.

\section{Model Predictive Control}

MPC is a widely spread technology in industry for control design [5]. A model of the control system is deducted and the constraints on the state variables are determined to be able to construct a valid state space. Based on this model and the constraints an optimization problem is determined. On every decision point the best decision is determined by taking the current state as initial state of the optimization problem. Based on the consequences of a decision and predictions of the change of external parameters, a forecast of the result of every decision is made. This leads to a prediction of the next state and again possible decisions can be determined and the results of these decisions can be predicted. The number of decision points taken into account is called the observed horizon.

In [6] the effect of the length of the observed horizon is analyzed. In literature it is observed that a larger horizon may lead to worse results, where large depends on the fluctuations in the optimization problem. A larger horizon can lead to continuously postponing taking profit, continuously make a less profitable decision to gain more profit in the future. The authors of [6] conclude that by using certain heuristics the performance of longer horizons improve and outperform shorter horizons.

MPC applied to the local control methodology: When $\mathrm{N}$ future states are taken into account, the observed horizon becomes $T=\{0,1, . ., N\}$. For every observed time interval there is a set of choices per device, every time interval the pools need to be balanced, etc. I.e, the algorithm described in the previous section is copied for every observed time interval. Therefore, an index $t \in T$ is added to every variable, e.g. $x_{d}$ becomes $x_{d, t}$ and (5) becomes:

$$
\sum_{d \in D} M_{d p, t} \times x_{d, t}=0 \forall p \in P, \forall t \in T
$$

This results in balance in every time interval and a valid chosen $x_{d}$ for every time interval for every device, i.e. per time interval the model is correct. However, not every state in time interval $t+1$ can be reached given the chosen state in time interval $t$, so constraints to guarantee a valid sequence of states are needed. This is done using the $c_{d, i, t}$ variables. For example, Figure 3 shows a state space for a freezer with on/off decision. In this case, the only valid preceding states of $S_{3,3}\left(c_{d, 3,3}\right)$ are $S_{2,2}$ and $S_{2,3}$. Therefore, the added constraint becomes $c_{d, 3,3}-c_{d, 2,2}-c_{d, 2,3} \leq 0$. So, $c_{d, 3,3}$ can only be chosen (become one) when one of the preceding states $c_{d, 2,2}$ or $c_{d, 2,3}$ is chosen. Only one of the two preceding states can be nonzero due to (8). Such a constraint needs to be added for every state where $t>0$.

Summarizing, by duplicating the model for each time period and adding constraints describing the possible state transitions an optimization model for a horizon can be achieved.

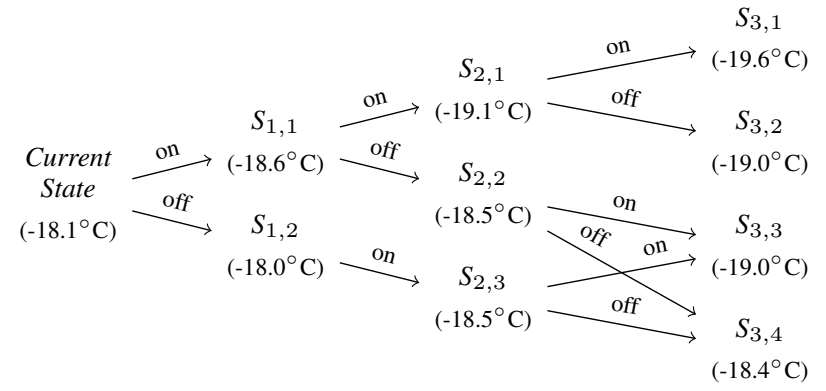

Fig. 3. $\mathrm{N}$ future states of a freezer

\section{V. (SMART) APPLIANCES}

The smart appliances implemented for the use-cases are a freezer, a heat buffer and a microCHP. The freezer has a state containing the internal temperature and whether it is switched on or off. The temperature $T$ has to be in the range $[-28,-18]^{\circ} \mathrm{C}$. The freezer has three different states:

- off - $2 \mathrm{~W}$ electricity consumption, the temperature increases $0.1{ }^{\circ} \mathrm{C}$ per 4 minutes

- on $\mathbf{T} \geq \mathbf{- 2 3}$ - $100 \mathrm{~W}$ electricity consumption, the temperature decreases with $0.5{ }^{\circ} \mathrm{C}$ per 4 minutes

- on $\mathbf{T}<-\mathbf{2 3}$ - $140 \mathrm{~W}$ electricity consumption, the temperature decreases with $0.5{ }^{\circ} \mathrm{C}$ per 4 minutes

Without optimization, the freezer is switched on when the temperature reaches $-18{ }^{\circ} \mathrm{C}$ and cools until the temperature is $-23{ }^{\circ} \mathrm{C}$. The $x_{d}$ has two valid choices in every state (on or off), except when the temperature is $-18{ }^{\circ} \mathrm{C}$ or $-28{ }^{\circ} \mathrm{C}$. The costs depend on the temperature and the current state (switching costs).

For the MPC extension, the model is used for the prediction of the temperature in future states. During the simulation pseudo-random variations are added to emulate prediction errors (e.g. people opening the freezer). Since in almost every state two possible next states are possible, the state space is organized in a tree structure as showed in Figure 3. These states can be calculated rather easily and these states are added and coupled using coupling constraints as described in the previous section.

For the realtime controller without MPC the heat buffer is modelled straightforward using a maximum capacity and the SoC (when heat flows to the buffer the SoC increases and when heat flows from the buffer the SoC decreases). The microCHP is modelled using a state machine with four states (off, starting running, stopping) with corresponding $x_{d}$ and $M_{d p}$.

When MPC is added, a heat buffer can have a lot of different next states from itself, $x_{d}$ can have a lot of different values, where the microCHP can only have two next states. However, in practice there are only a few possible next states for the heat buffer because of the required balance within pools and these states depend on the choices made for other devices (e.g. switching the microCHP on or off). Therefore, the status of the heat buffer is incorporated in the states of the house and 


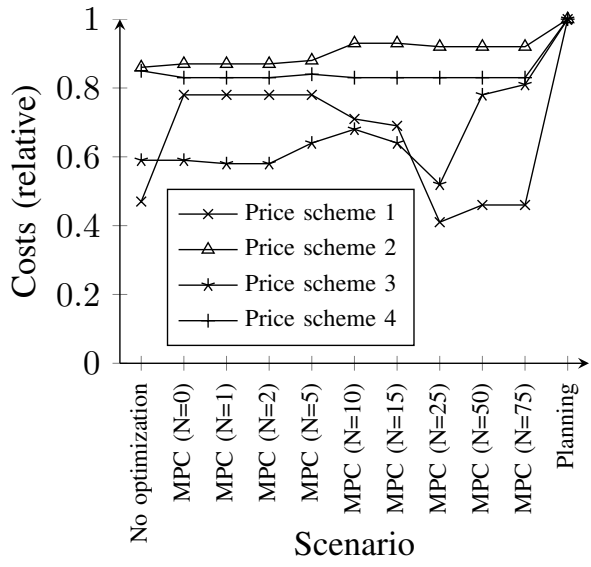

(a) Without variation

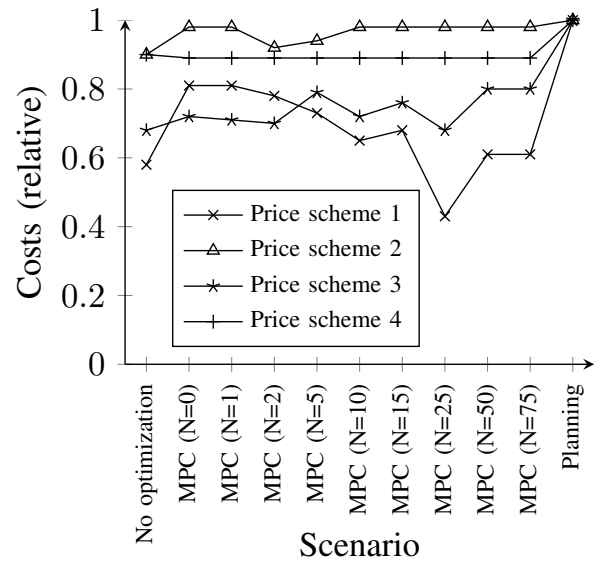

(b) With variation

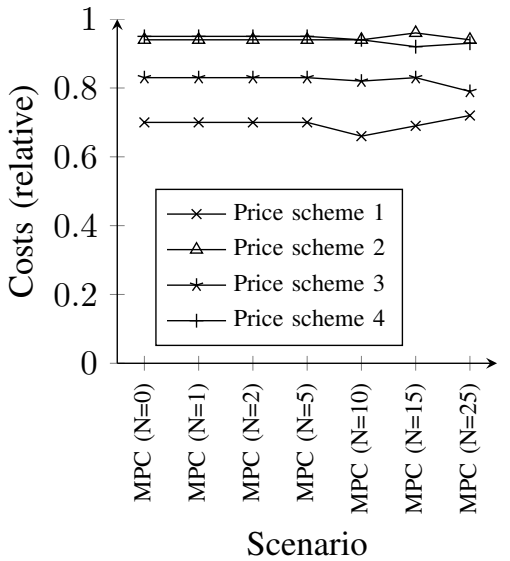

(c) Combination MPC and planning with variation

Fig. 4. Simulations MPC controlled freezer (relative from optimum, higher is better)

used to determine the valid states during the construction of the state space.

The MPC implementation for non-smart devices can be done in two ways: use the predicted behavior or leave them out of the observations. The advantage of leaving them out of the observations is that is simplifies the algorithms (and decreases computation time) and their behavior cannot be optimized anyway. However, some objectives require that the behavior is taken into account, e.g. peak shaving objectives. Therefore, we chose to incorporate the predicted (not manageable) behavior into the MPC.

\section{Simulations AND RESUlts}

This section first describes the use cases and then discusses the simulation results.

\section{A. Use Cases}

In the first use case the runtime of the freezer described in the previous section is optimized during one day with as objective to minimize the electricity costs. The time interval length is four minutes, so 360 time intervals are simulated. Four different pricing schemes are used with different levels of variation. The first scheme has five price levels based on a normal consumption pattern (minimal two hours the same price, high demand results in a high price), the second scheme has two levels (day and night), the third combines the first two and the last pricing scheme is based on the APX prices from April 15th 2009 (prices change per hour). Each pricing scheme is simulated using different scenarios:

- No optimization - Normal behavior based on temperature of the freezer.

- Realtime optimization - Only the last step of the three step methodology is used, the electricity prices are directly used as import prices for the algorithm.

- Realtime optimization with MPC - Previous scenario but with MPC (previous scenario can be seen as a special case of this scenario with a observed horizon of zero, $\mathrm{N}=0$ ).

- Planning - The three step methodology is used to generate steering signals, these steering signals are used by the last step (realtime optimization). The electricity prices are known in advance, the behavior of the freezer is predicted as described.

- Planning with MPC - Previous scenario, but MPC is added to the last step.

The results are compared based on the objective. More precisely, the average electricity price for the consumed electricity is compared. Furthermore, the number of starts are compared (how irregular is the resulting schedule, wearing of the device).

The second use case is an extension of the use case described in [8]. A neighborhood consisting of 39 houses is simulated, in each house a microCHP and a heat buffer is installed and the simulation horizon is one day (time interval length five minutes). In the described use case the objective was to reach a given production pattern (using the three-step methodology). From our database with real heat demand data of Dutch households, 39 heat profiles between Nov. 19, 2007 until Nov. 31, 2007 have been extracted and used as input for the simulations. Based on historical input data the heat demand is predicted and a planning determined. This planning is used as input for the realtime algorithm (last step) while the actual heat demand is used. Due to prediction errors the planning could not been reached, the imbalance was $77 \mathrm{kWh}$ (sum of absolute difference per time interval, SAD). For this paper we added MPC to the last step to analyst whether MPC is able to work around the prediction errors.

\section{B. Results use case 1}

The relative average costs from the simulated scenarios are given in Figure 4. A planning and perfect prediction result in a optimal solution (lowest costs), therefore all costs of the scenarios are expressed relative to the costs of these scenarios (higher is better). 
TABLE I

SIMULATION RESULTS 39 HOUSES WITH MICROCHP

\begin{tabular}{|c|c|c|}
\hline Observed horizon & Imbalance & Imbalance (relaxed) \\
\hline $\mathrm{N}=0$ & $75.9 \mathrm{kWh}$ & $75.9 \mathrm{kWh}$ \\
$\mathrm{N}=1$ & $73.3 \mathrm{kWh}$ & $73.3 \mathrm{kWh}$ \\
$\mathrm{N}=2$ & $71.6 \mathrm{kWh}$ & $71.6 \mathrm{kWh}$ \\
$\mathrm{N}=5$ & $73.6 \mathrm{kWh}$ & $72.5 \mathrm{kWh}$ \\
$\mathrm{N}=10$ & - & $74.6 \mathrm{kWh}$ \\
\hline
\end{tabular}

Figure 4(a) shows the results without variation (perfect predictions) for all scenarios except the combination of planning and MPC. Without variation the planning scenario always gives the best results. A $\mathrm{N}>10$ gives often worse results, the optimal value of $\mathrm{N}$ depends on the amount of fluctuation in the pricing scheme. Especially when the observed horizon is a bit longer than the fluctuations the results become worse. For slow or fast fluctuation (scheme 2 and 4 ) the value of $\mathrm{N}$ has almost no influence. Pricing scheme 3 has slower fluctuations than scheme 1 and therefore the negative influence is earlier gone. However the costs are not significant lower for higher value of $\mathrm{N}$, the number of starts decrease significantly when $\mathrm{N} \geq 5$, up to $70 \%$. Overall, without variation a value of $\mathrm{N}=5$ or $\mathrm{N}=10$ is the best solution.

Figure 4(b) shows the same scenarios but with variation (prediction errors). When variation is added, the planning still gives the best results. However, MPC scores relatively better than without variation, especially concerning the number of starts. For the rest the results follow the same trend as without variation.

Figure 4(c) shows the results for the simulations with variation and the scenario of a combination of planning and MPC. When MPC and planning are combined, the behavior increases significantly. If only planning is used (without MPC), prediction errors lead to very irregular behavior, resulting in a lot of starts. This is for example caused by steering signals to switch on the fridge while the temperature is already $-28^{\circ} \mathrm{C}$ (due to prediction errors). The freezer has to be switched off, a bit later the temperature is dropped and the freezer switched on again due to the steering signals for a short time. Combining the planning with MPC decreases the costs often only slightly, but the number of starts decrease significantly if $\mathrm{N}$ is chosen to be larger than 10 (more than 50\% in all scenarios).

The complexity of the ILP to be solved increases significantly when MPC is added and therefore the computational time increases as well. All simulations of this use case could be done within minutes, we expect that up to $\mathrm{N}=10$ the computational times are acceptable for an embedded solution. Therefore, for this use case adding MPC to the last step improves the results, the best observed horizon length is $\mathrm{N}=5$ or $\mathrm{N}=10$.

\section{Results use case 2}

The results of this use case are given in Table I. Due to a slightly improved cost function definition the results of the standard case $(\mathrm{N}=0)$ improved slightly in comparison to [8]. Adding MPC to the last step increases the results in this use case also, the imbalance reduces (the planning is followed better) and the behavior of the microcHP is less fluctuating (less short runs), just as with the freezer. But due to the buffer the state space becomes very large and for $\mathrm{N}>5$ solving the ILP takes too long (expected computational time of weeks). Studying the simulations in more detailed showed that determining an ILP solution is the bottleneck. Therefore, we relaxed the optimization problem by changing it into an Mixed Integer Programming allowing $x_{d, t}$ for $t>2$ to be non-integer. Since these states are based on predictions and these choices are not directly used this does not decrease the accuracy of the model. The results of these simulations are also given in Table I. Up to $\mathrm{N}=2$ the results are of course the same and for $\mathrm{N}=5$ they are a bit different. This is caused by the differences in the predicted states (due to the relaxation). For $\mathrm{N} \geq 10$ the imbalance start to increase again, which is probably caused by the effect of postponing the moment to 'take the profit'.

\section{CONCLUSIONS}

Extending the last step of our three-step methodology with MPC improves the results in both simulated use cases. Especially in combination with planning the MPC improves the results and mainly results in a more stable behavior of devices (without the fast on/off switching behavior). This more stable behavior can become important when our solution is used to maintain or even improve grid stability. The optimal length of the planning horizon depends on the frequency of the fluctuations, but $\mathrm{N}=5$ or $\mathrm{N}=10$ is often a good trade off between computational time and the results. Using an ILP leads to high computational time while the future states are predictions and subject to prediction errors. Therefore, relaxing the integer constraints for $\mathrm{t}>2$ in the ILP results in significantly lower computational times with the same results. However, better heuristics to decrease the computational time even more are required.

\section{REFERENCES}

[1] A. de Jong, E.-J. Bakker, J. Dam, and H. van Wolferen, "Technisch energie- en CO2-besparingspotentieel in Nederland (2010-2030)," Platform Nieuw Gas, p. 45, Juli 2006.

[2] A. Molderink, V. Bakker, M. Bosman, J. Hurink, and G. Smit, "A threestep methodology to improve domestic energy efficiency," in IEEE PES Conference on Innovative Smart Grid Technologies, 2010.

[3] V. Bakker, A. Molderink, M. G. C. Bosman, J. L. Hurink, and G. J. M. Smit, "On simulating the effect on the energy efficiency of smart grid technologies," in Proceedings of the 2010 Winter Simulation Conference submitted, 2010.

[4] A. Molderink, V. Bakker, M. Bosman, J. Hurink, and G. Smit, "Domestic energy management methodology for optimizing efficiency in smart grids," in IEEE conference on Power Technology. IEEE, 2009.

[5] A. Bemporad, "Model predictive control design: New trends and tools," in Proceedings of the 45th IEEE Conference on Decision \& Control, 2006.

[6] R. A. Russell and T. L. Urban, "Horizon extension for rolling production schedules: Length and accuracy requirements," International Journal of Production Economics, vol. 29, no. 1, pp. 111 - 122, 1993.

[7] S. Koch, F. Barcenas, and G. Andersson, "Using controllable thermal household appliances for wind forecast error reduction," in IFAC Conference on Control Methodologies and Technology for Energy Efficiency, 2010.

[8] A. Molderink, V. Bakker, M. G. C. Bosman, J. L. Hurink, and G. J. M. Smit, "Management and control of domestic smart grid technology submitted," IEEE Transactions on Smart Grid, 2010. 